\title{
Measuring Cellular Immunity in Coccidioidomycosis: The Time is Now
}

\author{
Neil M. Ampel • Richard F. Hector
}

Received: 2 February 2010/ Accepted: 2 February 2010/Published online: 16 February 2010

(C) Springer Science+Business Media B.V. 2010

In this issue of Mycopathologia, Castañón-Olivares et al. [1] report on coccidioidin skin-test antigens prepared in Mexico and their evaluation in subjects living in endemic and non-endemic areas of Mexico.

Coccidioidins were originally created early in the twentieth century, and the materials used in the report by Castañón-Olivares and coworkers are largely based on the coccidioidins developed and meticulously studied by Smith and colleagues in the middle of that century $[2,3]$. Coccidioidins are crude culture filtrates obtained from prolonged incubation and autolysis of the mycelial phase of the Coccidioides spp. fungus in liquid media. While the mycelial phase of the fungus exists in the soil, it is the spherule phase of the dimorphic fungus that persists in tissue, leading to the presumption that a skin-test reagent made from spherules would contain more relevant antigens. When the ability to cultivate spherules in vitro became available, a new skin test, spherulin, was developed. Data suggest that spherulin is more

\section{N. M. Ampel ( $\square)$}

Valley Fever Center for Excellence, University of Arizona, Southern Arizona Veterans Affairs Health Care System, Tucson, AZ, USA

e-mail: nampel@email.arizona.edu

\section{R. F. Hector}

Global Health Sciences, University of California at San Francisco, 50 Beale Street, Suite 1200, San Francisco, CA 94105, USA

e-mail: rhector@psg.ucsf.edu sensitive than mycelial-based coccidioidin [4], however, the utility of both preparations is recognized by clinicians. Indeed, from a wealth of literature, it is clear that a robust cellular immune response not only indicates that infection with Coccidioides has occurred but that the host has developed long-lived immunity [5]. The time-honored approach to measuring cellular immunity is the delayed-type hypersensitivity reaction manifested through skin testing using coccidioidins.

The irony of the subject report is that while the incidence of symptomatic coccidioidomycosis has been dramatically increasing over the last decade in California and Arizona [6,7], the coccidioidin skin test has not been commercially available in the United States for at least a decade. While the reasons for this are presumably driven by economic considerations in this era of "blockbuster" drugs and biologics, this does not diminish the test's clinical and epidemiological utility, particularly given the inability of modern medicine to significantly reduce the morbidity and mortality associated with this disease over the past decades.

Measurement of cellular immunity in coccidioidomycosis has several clinical uses. In endemic areas, a negative assay would indicate that the individual is not infected and not immune. The subsequent development of pneumonia could suggest the possibility of primary pulmonary coccidioidomycosis. Alternatively, a previously positive test would preclude this diagnosis. Measurement of cellular immunity may 
also be useful in determining length of antifungal therapy in those with coccidioidomycosis requiring treatment. A study by Oldfield and colleagues suggests that in patients with complicated coccidioidomycosis, the failure to develop a positive coccidioidin skin test during the course of therapy is associated with a higher frequency of relapse when therapy is discontinued [8]. Finally, the test could be used to provide data on the prevalence of coccidioidal infection in certain geographic regions. For example, Larwood has reported on the decline of skin-test positivity in the highly endemic southern San Joaquin Valley over a 58-year period using data when the skin test was still available [9]. Knowing the current prevalence of infection in a specific geographic area would allow an estimate of the number of persons susceptible to infection and disease.

Coccidioidal cellular immunity can be determined by other means, including measuring the in vitro release of cytokines, such as interferon- $\gamma$, in response to incubation of blood with coccidioidal antigens. We have demonstrated the feasibility of this approach and have shown, in a small study, equivalence with mycelial-based coccidioidin [10]. This test is analogous to the currently marketed assays for tuberculosis and could be commercially developed.

The ability to measure cellular immunity is an important tool in the management of coccidioidomycosis. Thanks to Castañón-Olivares and colleagues, patients living in the endemic regions of Mexico will have continued access to it. The endemic regions of the United States deserve no less.

\section{References}

1. Castañón-Olivares L, Laniado-Laborin R, Concepcion T, Muñoz-Hernández B, Aroch-Calderón A, Aranda-Uribe IS, et al. Clinical comparison of two Mexican coccidioidins. Mycopathologia. 2010;in press.

2. Smith CE, Beard RR, Whiting EG, Rosenberger HG. Varieties of coccidioidal infection in relation to the epidemiology and control of the diseases. Am J Public Health. 1946;36:1394-402.

3. Smith CE, Whiting EG, Baker EE, Rosenberger HG, Beard R, Saito MT. The use of coccidioidin. Am Rev Tuberc. 1948;57:330-60.

4. Stevens DA, Levine HB, TenEyck DR. Dermal sensitivity to different doses of spherulin and coccidioidin. Chest. 1974;65(5):530-3.

5. Ampel NM. Measurement of cellular immunity in human coccidioidomycosis. Mycopathologia. 2003;156(4):24762.

6. CDC. Increase in Coccidioidomycosis-California, 20002007. MMWR Morb Mortal Wkly Rep. 2009;58(5):105-9.

7. Park BJ, Sigel K, Vaz V, Vaz V, Komatsu K, McRill C, et al. An epidemic of coccidioidomycosis in Arizona associated with climatic changes, 1998-2001. J Infect Dis. 2005;191(11):1981-7.

8. Oldfield EC III, Bone WD, Martin CR, Gray GC, Olson P, Schillaci RF. Prediction of relapse after treatment of coccidioidomycosis. Clin Infect Dis. 1997;25(5):1205-10.

9. Larwood TR. Coccidioidin skin testing in Kern County, California: decrease in infection rate over 58 years. Clin Infect Dis. 2000;30(3):612-3.

10. Ampel NM, Hector RF, Lindan CP, Rutherford GW. An archived lot of coccidioidin induces specific coccidioidal delayed-type hypersensitivity and correlates with in vitro assays of coccidioidal cellular immune response. Mycopathologia. 2006;161(2):67-72. 